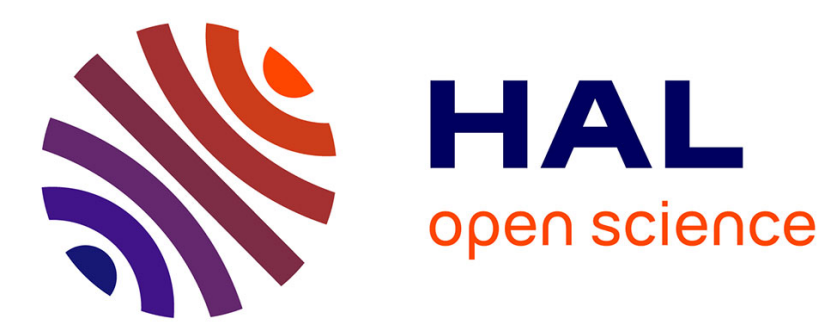

\title{
Stability analysis and gain synthesis for Lipschitz non linear systems under dynamic event triggered sampling
}

Lucien Etienne, Stefano Di Gennaro, Jean-Pierre Barbot

\section{To cite this version:}

Lucien Etienne, Stefano Di Gennaro, Jean-Pierre Barbot. Stability analysis and gain synthesis for Lipschitz non linear systems under dynamic event triggered sampling. European Control Conference, Jun 2018, Lymassol, Cyprus. hal-01915514

\author{
HAL Id: hal-01915514 \\ https://hal.science/hal-01915514
}

Submitted on 7 Nov 2018

HAL is a multi-disciplinary open access archive for the deposit and dissemination of scientific research documents, whether they are published or not. The documents may come from teaching and research institutions in France or abroad, or from public or private research centers.
L'archive ouverte pluridisciplinaire HAL, est destinée au dépôt et à la diffusion de documents scientifiques de niveau recherche, publiés ou non, émanant des établissements d'enseignement et de recherche français ou étrangers, des laboratoires publics ou privés. 


\title{
Stability analysis and gain synthesis for Lipschitz non linear systems under dynamic event triggered sampling
}

\author{
L. Etienne, S. Di Gennaro, and J.-P. Barbot
}

\begin{abstract}
In this paper, we investigate the stabilization of a Lipschitz non linear plant under the assumption of dynamic event triggered sampling. Sufficient conditions, making use of a hybrid Lyapunov function and convex embedding, are given to guarantee the existence of a triggering mechanism, leading to asymptotic stability. Both gain analysis and gain synthesis are considered.
\end{abstract}

\section{INTRODUCTION}

In modern control systems, the use of the digital technology is pervasive. The control task consists of the sampling of the plant outputs, the computation, and the implementation of the actuator signals (e.g. zero order hold, P.W.M., Gaz jet). The classic way is to sample in a periodic fashion, thus allowing the closed-loop system to be analysed on the basis of sampled-data systems, see [2].

In the past decade a new methodology has been developed where the controller is not sampled periodically (i.e. with a time-triggered policy), but rather sampled when some information have sufficiently changed (i.e. externally an event trigger policy and internally self-trigger policy). A great number of works deal with this subject, see for instence [1], [7], [8], [11], [16],[18], [20], [21], [22], see also [12] for an introduction to this topic.

In recent year the concept of dynamic event trigger [9] has been proposed in order to further reduce communication (see also [19] and [5]). The main idea of dynamic event trigger is to create a new dynamic including the static event triggering policy acting as a 'filter' to the static event triggering criterion.

The main objective of this paper is to address the problem of dynamic event trigger stabilization of a Lipschitz nonlinear system. Namely we aim at providing numerically tractable sufficient conditions to synthesize a dynamic event triggering policy. The usual methodology to address stability is to consider a gain obtained from the continuous time model and to show that the resulting event triggered system is stable, this emulation (of the continuous model) methodology will be first considered and sufficient conditions for known gain analysis will be considered. Then, sufficient conditions for gain synthesis directly based on the hybrid model will be given, thus avoiding an emulation based approach.

The paper is organized as follows: In Section II we set the problem and introduce the hybrid framework ([10]) that will be used to prove our results. In Section III we give sufficient conditions in terms of an LMI feasibility problem in order to ensure stability of the system under consideration. Then sufficient conditions for simultaneous dynamic event trigger policy synthesis and gain synthesis are given. In Section IV two examples are considered. Finally, in Section VI we give some concluding remarks.

\section{Notations}

- For a vector or a matrix $v, v^{\prime}$ denotes its transpose.

- We define for a matrix $A, \operatorname{He}(A):=A+A^{\prime}$.

- $\mathbb{R}_{>0}$ corresponds to the non-negative real numbers.

- Given $p \in \mathbb{N}$, the set $\Delta_{p}$ denotes the unit simplex,

$$
\Delta_{p}:=\left\{\lambda \in \mathbb{R}_{\geq 0}^{p}: \sum_{i=1}^{p} \lambda_{i}=1\right\} .
$$

- For a set of vector $v_{i} \in \mathbb{R}^{n}, i=1, \ldots p, \overline{\operatorname{Cov}}\left\{v_{i}\right\}_{i \in\{1 \ldots p\}}$ denotes its closed convex hull

$$
\overline{\operatorname{Cov}}\left\{v_{i}\right\}_{i \in\{1 \ldots p\}}=\left\{V: \exists \lambda \in \Delta_{p}, \sum_{i=1}^{p} \lambda_{i} v_{i}=V\right\} .
$$

- $\mathbb{R}^{+}$denotes the set of non-negative real numbers (including 0 ).

- $\mathbb{S}$ denotes the set of symmetric matrices while $\mathbb{S}^{+}$denotes the set of positive definite symmetric matrices

- For a $M \in \mathbb{S}, \lambda_{\min }(M)$ and $\lambda_{\max }(M)$ denote its smallest and largest eigenvalues, respectively.

Lucien Etienne is with the departement of Informatic and automatic, Ecole des mines de Douai, 941 Rue Charles Bourseul, 59500 Douai. E.mail: lucien.etiennedimt-lille-douai.fr

Stefano Di Gennaro are with the Department of Information Engineering, Computer Science and Mathematics, and with the Center of Excellence DEWS, University of L'Aquila, Via Vetoio, Loc. Coppito, 67100 L'Aquila, Italy. E.mail: stefano.digennaro@univaq. it

Jean-Pierre Barbot is with the Quartz Laboratory EA 7393, 6 Avenue du Ponçeau, 95014 Cergy Pontoise Cedex E.mail: barbot@ensea.fr. 
- For two positive definite matrices (resp positive semidefinite) $P$ and $Q$ we write $P>Q$ if $P-Q$ is positive definite (resp $P \geq Q$ if $P-Q$ is positive semidefinite).

- $|\cdot|$ denotes the euclidean norm and $\|.\|_{P}$ is the norm induced by $P \in \mathbb{S}^{+}$.

- a function $\rho: \mathbb{R} \rightarrow \mathbb{R}$ is called positive definite (later abbreviated $\rho \in \mathcal{P} \mathcal{D}$ ) if $\forall x>0, \rho>0$

\section{Problem Statement}

Considering the following continuous time system with piecewise constant control

$$
\begin{aligned}
\dot{x}(t) & =f(x(t)) x(t)+g(x(t)) u(t) \\
u(t) & =K x\left(t_{k}\right), \forall t \in\left[t_{k}, t_{k+1}\right),
\end{aligned}
$$

with $\forall x \in \mathbb{R}^{n}, u(t) \in \mathbb{R}^{m}$.

Hereinafter, we will use the following assumption.

Assumption 1: The previous system can be written

$$
\begin{aligned}
& \dot{x}(t)=\sum_{i=1}^{p} \lambda_{i}(x(t)) A_{i} x(t)+\sum_{j=1}^{q} \mu_{j}(x(t)) B_{j} u(t), \\
& u(t)=K x\left(t_{k}\right), \forall t \in\left[t_{k}, t_{k+1}\right),
\end{aligned}
$$

with $\lambda(x)=\left(\lambda_{1}(x), \ldots, \lambda_{p}(x)\right) \in \Delta_{p}, \mu(x)=\left(\mu_{1}(x), \ldots, \mu_{q}(x)\right) \in \Delta_{q}$. Assumption 1 imply that the system is globally Lipschitz.

In what follows the sampling sequence $\left(t_{k}\right)_{k \in \mathbb{N}}$ is generated by an underlying dynamic event triggering mechanism given by

$$
\begin{aligned}
& \tau\left(t_{k}\right)=0 \\
& \dot{\tau}(t)=\sigma x(t)^{\prime} P x-\left(x(t)-x\left(t_{k}\right)\right)^{\prime} P\left(x(t)-x\left(t_{k}\right)\right), \\
& t_{k+1}:=\min \left\{t>t_{k} \text { s.t. }(\tau(t)<0) \vee\left(\tau(t)=x(t)^{\prime} Q x(t)\right)\right\} .
\end{aligned}
$$

$Q, P \in \mathbb{S}^{+}$are matrices to be chosen and $\sigma>0$ is a tuning parameter.

Remark 1: Note that a classic static event triggering policy considers the first time instant where $\sigma\|x(t)\|_{P}^{2}-\| x(t)-$ $x\left(t_{k}\right) \|_{P}^{2}$ is greater than zero.

By definition of $\dot{\tau}$, we have:

$$
\tau(t)=\int_{t_{k}}^{t} \sigma\|x(s)\|_{P}^{2}-\left\|x(s)-x\left(t_{k}\right)\right\|_{P}^{2} d s .
$$

Introducing this integral enables us to sample only when the static triggering condition is violated for a sufficiently large amount of time. Here $\tau$ can be seen as a "stability margin". Therefore, the sampling condition $(\tau<0)$ is introduce to ensure that the so called stability margin is non-negative. The condition $\left(\tau=x^{\prime} Q x\right)$ ensures that $\tau$ is not too big with respect to $x$ and is used both for internal stability and to avoid absence of sampling for a (non-asymptotically) stable system.

Remark 2: At time $t_{k}$ it holds that $x-x\left(t_{k}\right)=0$, hence $\dot{\tau}>0$.

\section{A. Generalities on hybrid systems}

Next, we will embed ${ }^{1}$ (1) system into the hybrid framework described for example in [10]. Consider a hybrid system

$$
\mathcal{H}: \begin{cases}\xi \in C_{\mathcal{H}}, & \dot{\xi} \in F_{\mathcal{H}}(\xi), \\ \xi \in D_{\mathcal{H}}, & \xi^{+} \in G_{\mathcal{H}}(\xi) .\end{cases}
$$

Roughly speaking, while $\xi$ belongs to $C_{\mathcal{H}}$, the state flows according to a differential inclusion characterized by a set-valued mapping $F_{\mathcal{H}}$. When $\xi$ belongs to $D_{\mathcal{H}}$, the state jumps according to a discrete dynamic defined by $G_{\mathcal{H}}$. In what follows we will use the concepts and notations from [10].

Definition 1 (Candidate Lyapunov function): A function $V: \operatorname{dom} V \rightarrow \mathbb{R}$ is said to be a candidate Lyapunov function for the hybrid system $\mathcal{H}$ if the following conditions hold:

1. $\overline{C_{\mathcal{H}}} \cup D_{\mathcal{H}} \cup G_{\mathcal{H}}\left(D_{\mathcal{H}}\right) \subset \operatorname{dom} V$;

2. $V$ is continuously differentiable on an open set containing $\overline{C_{\mathcal{H}}}$, where $\overline{C_{\mathcal{H}}}$ denotes the closure of $C_{\mathcal{H}}$.

We recall now the Theorem 3.18 of [10]:

\footnotetext{
${ }^{1}$ In this context embed means that the system (1) will be studied trough a hybrid dynamical system containing more solutions. It is however sufficient that to any solution of (1) corresponds a solution to the hybrid system.
} 
Theorem 1 (Sufficient conditions for $U G p A S$ ): Let $\mathcal{H}$ be a hybrid system and let $\mathcal{A} \subset \mathbb{R}^{n}$ be closed. If $V$ is a Lyapunov function candidate for $\mathcal{H}$ and there exist $\alpha_{1}, \alpha_{2} \in \mathcal{K}_{\infty}$, and a continuous $\rho \in \mathcal{P} \mathcal{D}$ such that:

$$
\begin{gathered}
\forall \xi \in C_{\mathcal{H}} \cup D_{\mathcal{H}} \cup G_{\mathcal{H}}\left(D_{\mathcal{H}}\right) \\
\alpha_{1}\left(|\xi|_{\mathcal{A}}\right) \leq V(\xi) \leq \alpha_{2}\left(|\xi|_{\mathcal{A}}\right), \\
\forall \xi \in C_{\mathcal{H}}, f \in F_{\mathcal{H}}(\xi),\langle\nabla V(\xi), f\rangle \leq-\rho\left(|\xi|_{\mathcal{A}}\right), \\
\forall \xi \in D_{\mathcal{H}}, g \in G_{\mathcal{H}}(\xi), V(g)-V(\xi) \leq 0 .
\end{gathered}
$$

If, for each $r>0$, there exists $\gamma_{r} \in \mathcal{K}_{\infty}, N_{r} \geq 0$ such that for every solution $\phi$ to $\mathcal{H}$

$$
\begin{array}{r}
|\phi(0,0)|_{\mathcal{A}} \in(0, r],(t, j) \in \operatorname{dom} \phi, \\
t+j \geq T \text { imply } t \geq \gamma_{r}(T)-N_{r}
\end{array}
$$

then $\mathcal{A}$ is UGpAs for $\mathcal{H}$.

Remark 3: This theorem can be interpreted as follows: While (5a) ensure positive definiteness of the hybrid Lyapunov function, (5b) ensure strict decrease during flows and (5c) ensures absence of increase during jump. The last conditions, $(5 \mathrm{~d})$, ensures that the system "flows enough" in the sense that for $(t, j)$ in $d o m \phi, t$ cannot be to small with respect to $j$.

\section{B. Hybrid system representation}

Next we will use the proposed formalism in order to conduct the stability analysis of a system subject to dynamical event trigger. In the proposed formalism the system can be written in the following way:

and

$$
\left.\begin{array}{l}
\dot{x} \in \overline{\operatorname{Cov}}\left\{A_{i} x\right\}_{i \in\{1 \ldots p\}}+\overline{\operatorname{Cov}}\left\{B_{j} K z\right\}_{j \in\{1 \ldots q\}} \\
\dot{z}=0 \\
\dot{\tau}=\sigma\|x\|_{P}^{2}-\|x-z\|_{P}^{2}
\end{array}\right\}\left(\begin{array}{c}
x \\
z \\
\tau
\end{array}\right) \in C_{\mathcal{H}}
$$

$$
\left.\begin{array}{l}
x^{+}=x \\
z^{+}=x \\
\tau^{+}=0
\end{array}\right\}\left(\begin{array}{l}
x \\
z \\
\tau
\end{array}\right) \in D_{\mathcal{H}} .
$$

Here (6) corresponds to the system dynamic in between sampling times, i.e. to (1), while (7) describes the impulsive dynamics i.e. when $u(t)$ changes value. The system state is augmented to include a sampled value of $x$ written $z$ and a "dynamic event generator" $\tau$. Defining the extended state $\xi:=\left(x,{ }^{\prime} z^{\prime}, \tau\right)^{\prime}$, the hybrid system (6), (7) can be rewritten in the form (4) with

$$
C_{\mathcal{H}}=\left\{\xi \in \mathbb{R}^{2 n+1} \mid(x, z) \in \mathbb{R}^{2 n},\left(x^{\prime} Q x>\tau\right) \wedge(\tau \geq 0)\right\} ;
$$

the flow map

$$
F_{\mathcal{H}}(\xi)=\left(\begin{array}{c}
\overline{\operatorname{Cov}}\left\{A_{i} x\right\}_{i \in\{1 \ldots p\}}+\overline{\operatorname{Cov}}\left\{B_{j} K z\right\}_{j \in\{1 \ldots q\}} \\
0 \\
\sigma^{2}\|x\|_{P}^{2}-\|x-z\|_{P}^{2}
\end{array}\right)
$$

for $\xi$ in $C_{\mathcal{H}}$ and $F_{\mathcal{H}}(\xi)=\emptyset$ elsewhere; the jump set

$$
D_{\mathcal{H}}=\left\{\xi \in \mathbb{R}^{2 n+1} \mid,(x, z) \in \mathbb{R}^{2 n},\left(x^{\prime} Q x=\tau\right) \vee(\tau<0)\right\} ;
$$

and the jump map

$$
G_{\mathcal{H}}(\xi)=\left(\begin{array}{ccc}
I & 0 & 0 \\
I & 0 & 0 \\
0 & 0 & 0
\end{array}\right) \xi
$$

when $\xi$ in $D_{\mathcal{H}}$ and $G_{\mathcal{H}}(\xi)=\emptyset$ elsewhere.

Remark 4: Consider a solution $w$ of system (1) and $\left(t_{k}\right)_{k \in \mathbb{N}}$ defined by (3). Given $E=\cup_{k=0}^{\infty}\left(\left[t_{k}, t_{k+1}\right], k\right)$, define the hybrid arc $\xi: E \rightarrow \mathbb{R}^{n+2}$ as follows

$$
\xi(t, k)=\left(w(t)^{\prime}, w\left(t_{k}\right)^{\prime}, \tau(t)\right)^{\prime}, t \in\left[t_{k}, t_{k+1}\right),
$$

and

$$
\xi\left(t_{k+1}, k\right)=\lim _{t \rightarrow t_{k+1}, t<t_{k+1}}\left(w(t)^{\prime}, w^{\prime}\left(t_{k}\right), 0\right)^{\prime} .
$$

Using the description (6), (7), one can see that $\xi$ is a complete solution to the hybrid system (4) with data $C_{\mathcal{H}}, F_{\mathcal{H}}, D_{\mathcal{H}}, G_{\mathcal{H}}$ as in (8)-(11). 
Remark 5: System (4), (8)-(11) has more solution than system (1), (3) however from the previous remark all solution of (1), (3) are solutions of (4), (8)-(11)

For system (4), (8)-(11) we consider UGpAS with respect to the set

$$
\mathcal{A}=\left\{\xi \in C_{\mathcal{H}} \cup D_{\mathcal{H}} \mid x=0\right\} .
$$

The problem addressed here is formalized as follows:

Problem: Consider system (4), (8)-(11). Show that the set $\mathcal{A}$ is UGpAS.

Remark 6: Since all solutions of (8)-(11) corresponding to a solution of (1), (3) are complete, then the UGpAS of the set $\mathcal{A}$ for the hybrid system (4), (8)-(11) implies that the equilibrium $x=0$ of (1) is globally asymptotically stable. Note that for any $\xi \in C_{\mathcal{H}} \cup D_{\mathcal{H}},|\xi|_{\mathcal{A}}=|x|$. From Remark 1 and Definition (??), UGpAS for the set $\mathcal{A}$ defined in (14) for (4), (8)-(11) implies for (1) that there exists a $\mathcal{K}_{\infty}$ function $\alpha$ such that

$$
|x(t)| \leq \alpha(|x(0)|), \forall t \geq 0 .
$$

Furthermore, for each $\varepsilon>0$ and $r>0$ there exists $\tilde{T}>0$ such that, if $|x(0)| \leq r$, then $|x(t)| \leq \varepsilon$ for all $t \geq \tilde{T}$, which implies that $\lim _{t \rightarrow \infty} x(t)=0$.This corresponds to the classical definition of asymptotic stability of system (1), (3)

\section{MAIN RESULTS}

First we provide UGpAS analysis conditions for system (4), (8)-(11). The proposed conditions are based on the existence of a hybrid Lyapunov function ${ }^{2}$ of the form $V(\xi)=x^{\prime} M x+\tau$ satisfying the assumptions given by Definition 1 and Theorem 1 .

Theorem 2: Consider the hybrid system (4), (8)-(11), $M, P \in \mathbb{S}^{+}$, and the following set of LMIs :

$$
\mathcal{M}_{i, j}:=\left(\begin{array}{cc}
\left(\sigma^{2}-1\right) P+H e\left(M A_{i}\right) & M B_{j} K+P \\
\star & -P
\end{array}\right)<0
$$

If the LMIs (15) is verified, then the set $\mathcal{A}$ defined in (14) is UGpAS and solution of (4), (8)-(11) does not posses Zeno behaviour.

Proof: Here, we show that the set $\mathcal{A}$ is UGpAS using the Theorem 1.

Define $V(\xi)=x^{\prime} M x+\tau$. First $V$ is a candidate hybrid Lyapunov function in the sense of Definition 1 since $\overline{C_{\mathcal{H}}} \cup D_{\mathcal{H}} \cup$ $G_{\mathcal{H}}\left(D_{\mathcal{H}}\right) \subset \operatorname{dom} V$ and $V$ is continuously differentiable on an open set containing $\overline{C_{\mathcal{H}}}$.

One has $\forall \xi \in C_{\mathcal{H}} \cup D_{\mathcal{H}} \cup G_{\mathcal{H}}\left(D_{\mathcal{H}}\right) \lambda_{\min }(M)|x|^{2}=\lambda_{\min }(M)|\xi|_{\mathcal{A}}^{2} \leq V(\xi) \leq \lambda_{\max }(M)|\xi|_{\mathcal{A}}^{2}=\left(\lambda_{\max }(Q)\right)+\lambda_{\max }(M)|x|^{2}$,

Therefore (5a) is satisfied.

(i) Minimal time between events

Note that after the first reset of $\tau$ we have that $\tau\left(t_{1}, 1\right)=0, z\left(t_{1}, 1\right)=x\left(t_{1}, 1\right)$ We will now show that after the first reset there exist a time $t^{*}$ where the system flows (i.e. $\xi \in C_{\mathcal{H}}$ ). Denoting $e=x-z$, we consider the evolution of $e P e / x P x$ and shows that there is a minimal time before $e P e / x P x$ reaches $\sigma^{2}$. Note that $\dot{x}=\dot{e}$, and $|x| \leq L(|x|+|e|)$ with $L=\max _{i, j}\left(\left|B_{j} K\right|,\left|A_{i}+B_{j} K\right|\right)$.

Considering

$$
\begin{gathered}
\frac{d}{d t} \frac{|e|^{2}}{|x|^{2}} \leq \frac{|\dot{x}|}{|x|}\left(\frac{|e||x|^{2}+|x||e|^{2}}{|x|^{3}}\right), \\
\frac{d}{d t} \frac{|e|^{2}}{|x|^{2}} \leq 2 L\left(1+\frac{|e|}{|x|}\right)^{2} \frac{|e|}{|x|},
\end{gathered}
$$

using the comparison lemma with a function $y(0)=0, \dot{y}=L(1+y)^{2}$, we conclude that $\frac{|e(t)|}{|x(t)|} \leq \frac{L t}{1-L t}$. Thus

$$
\frac{e(t)^{\prime} P e(t) \mid}{x(t)^{\prime} P x(t)} \leq \frac{\lambda_{\max }(P)}{\lambda_{\min }(P)}\left(\frac{L t}{1-L t}\right)^{2}
$$

Hence for all time $t \leq t_{\min }^{1}:=\frac{\sigma}{L\left(\sqrt{\frac{\lambda_{\max }(P)}{\lambda_{\min }(P)}}+\sigma\right)}$ It holds that $\dot{\tau} \geq 0$.

We will also show that (after the first reset) $\tau<x^{\prime} Q x$ for at least a fixed amount of time. Note first that there exist $\bar{L}>0$ such that $\dot{\tau}<\sigma^{2} \bar{L}|x|^{2}, \frac{d}{d t}|x| \leq \bar{L}|x|+|e|$ Considering $\frac{\tau}{|x|^{2}}$ one works out

$$
\frac{d}{d t}\left(\frac{\tau}{|x|^{2}}\right) \leq \frac{\dot{\tau}|x|^{2}+\bar{L}|x|(|x|+|e|) \tau}{|x|^{4}}
$$

\footnotetext{
${ }^{2}$ A hybrid Lyapunov function similar to $V(\xi)=x^{\prime} M x+\tau$ as been proposed in [19] to study stability of dynamic event triggered systems for linear systems with limited sensors information.
} 
We have also established that $|e(t)| \leq \frac{L t}{1-L t}|x(t)|$ Denoting $G(t)=\frac{L t}{1-L t}$ we have that

$$
\frac{d}{d t}\left(\frac{\tau}{|x|^{2}}\right) \leq \bar{L}\left(\sigma^{2}+(1+G(t))\left(\frac{\tau}{|x|^{2}}\right)\right)
$$

Using the comparison lemma with $y(0)=0, \dot{y}=\bar{L}\left(\sigma^{2}+(1+G(t)) y\right)$, one can prove that there exist $t_{\text {min }}^{2}$ such that

$$
\frac{\tau(t)}{\lambda_{\max }(Q) x^{\prime} Q x} \leq \frac{\tau(t)}{|x|^{2}}<1
$$

so for all $t \leq t_{\min }^{2}, \tau(t) \leq x^{\prime} Q x<1$.

We can conclude that between two consecutive jumps (except potentially the first one) at least $t^{*}$ amount of time elapse with

$$
t^{*}=\min \left(t_{\min }^{1}, t_{\min }^{2}\right)
$$

Hence considering a solution $\phi$, to $\mathcal{H}$ with $(t, j) \in \operatorname{dom} \phi$, one has that $j \leq 2+t / t^{*}$ (indeed after initialization the time between consecutive jumps is lower bounded by $t^{*}$ ) Thus

$$
t+j \geq T \Rightarrow t \geq\left(1+1 / t^{*}\right)^{-1} T-2\left(1+1 / t^{*}\right)^{-1} .
$$

Therefore the properties (5d) is verified for (4), (8)-(11).

(ii) Conditions during flow $\left(\xi \in C_{\mathcal{H}}\right)$ :

Note that $\forall \xi \in C_{\mathcal{H}}, f \in F_{\mathcal{H}}(\xi)$

$$
\langle\nabla V, f\rangle \in \overline{\operatorname{Cov}}\left\{\alpha_{i}\right\}_{i \in\{1 \ldots p\}}+\overline{\operatorname{Cov}}\left\{\beta_{j}\right\}_{j \in\{1 \ldots q\}}
$$

with $\alpha_{i}=x^{\prime} M A_{i} x+x^{\prime} A_{i}^{\prime} M x$ and $\beta_{j}=z^{\prime}\left(B_{j} K\right)^{\prime} M x+x^{\prime} M B_{j} K z+\dot{\tau}$, which can be expressed as

$$
\sum_{i=1}^{p} \lambda_{i}(x) \sum_{j=1}^{q} \mu_{j}(x)\left(x^{\prime}, z^{\prime}\right) \mathcal{M}_{i j}\left(\begin{array}{l}
x \\
z
\end{array}\right)
$$

Since the LMIs (15) are strict, there exists a sufficiently small $\varepsilon>0$ such that

$$
\mathcal{M}_{i j}<-\varepsilon I
$$

Thus $\forall \xi \in C_{\mathcal{H}}, \forall f \in \mathcal{F}_{\mathcal{H}}(\xi)$,

$$
\langle\nabla V, f\rangle=\sum_{i=1}^{p} \lambda_{i}(x) \sum_{j=1}^{q} \mu_{j}(x)\left(x^{\prime}, z^{\prime}\right) \mathcal{M}_{i j}\left(\begin{array}{c}
x \\
z
\end{array}\right) \leq-\varepsilon|\xi|_{\mathcal{A}}^{2},
$$

that is $(5 b)$ is verified.

(iii) Conditions during jump $\left(\xi \in D_{\mathcal{H}}\right)$ :

From the fact that $\forall \xi \in D_{\mathcal{H}}, \tau \geq 0$ And $\tau^{+}=0$. Since $\forall g \in G_{\mathcal{H}}(\xi), V(g)-V(\xi)=-\tau$, the hybrid Lyapunov function is (non strictly) decreasing at jumping time. All the condition of Theorem 2 are verified thus the set $\mathcal{A}$ is UGpAS.

Remark 7: Note that the proposed framework allows to compute the norm $P$ for a fixed $\sigma$ (if the LMI (15) is feasible) however to state the problem as an LMI feasibility problem either $P$ or $\sigma$ has to be a priori fixed.

Remark 8: The matrix $Q$ plays almost no role in the proof except to state $V(\xi) \leq \lambda_{\max }(M)|\xi|_{\mathcal{A}}^{2}=\left(\lambda_{\max }(Q)\right)+$ $\lambda_{\max }(M)|x|^{2}$. As such $Q \in \mathbb{S}^{+}$can be chosen arbitrarily without impacting the proof of stability, however the choice of $Q$ may impact the rate of convergence and the sampling occurrence.

Remark 9: In the description of (1) no controllability hypothesis were made. However stabilizability of every $A_{i}$ is a necessary condition for (15) to be feasible. Indeed, multiplying (15) on the left by $(I, I)$ and on the right by $(I, I)^{\prime}$ one finds out that

$$
\forall i \in\{1, \cdots, p\}, \forall j \in\{1, \cdots, q\} \sigma^{2} P+H e\left(P\left(A_{i}+B_{j} K\right)<0 .\right.
$$

This inequality implies the stabilitzability of each linear system of the form $\dot{x}=A_{i} x+B_{j} u$. 


\section{A. LMI conditions for controller synthesis}

Until know it has been assumed that the control gain $K$ implemented along with the dynamic triggering condition (3) was a priori known. The resulting aproach is called emulation based as it assumes knowledge of a controller computed using the continuous time model. Next, using the proposed framework it is possible to find a control gain $K$ alongside the matrix $P$.

Theorem 3: Consider the hybrid system (4), (8)-(11), $S, \bar{P} \in \mathbb{S}^{+}, L \in \mathbb{R}^{m \times n}$ and the following set of LMIs is verified $\forall i \in\{1, \cdots, M\}$

$$
\mathcal{S}_{i, j}:=\left(\begin{array}{cc}
\left(\sigma^{2}-1\right) \bar{P}+S A_{i}^{\prime}+A_{i} S & B_{j} L+\bar{P} \\
\star & -\bar{P}
\end{array}\right)<0 .
$$

If the LMIs (15) is verified, then the set $\mathcal{A}$ defined in (14) is UGpAS with $K=L S^{-1}$ and $P=S^{-1} \bar{P} S^{-1}$ and solution of (4), (8)-(11) does not posses Zeno behavior.

Proof: Assume that the set of LMIs (16) is feasible. Computing $\left[\begin{array}{ll}S & 0 \\ 0 & S\end{array}\right]^{-1} \mathcal{S}_{i, j}\left[\begin{array}{ll}S & 0 \\ 0 & S\end{array}\right]^{-1}$

One obtains

$$
\left(\begin{array}{cc}
\left(\sigma^{2}-1\right) S^{-1} \bar{P} S^{-1}+H e\left(A_{i}^{\prime} S^{-1}\right) & S^{-1} B_{j} L S^{-1}+S^{-1} \bar{P} S^{-1} \\
\star & -S^{-1} P S^{-1}
\end{array}\right),
$$

by writing $K=L S^{-1}, M=S^{-1}$ and $P=S^{-1} \bar{P} S^{-1}$. We have that

$$
\left(\begin{array}{cc}
\left(\sigma^{2}-1\right) P+H e\left(M A_{i}\right) & M B_{j} K+P \\
\star & -P
\end{array}\right)<0
$$

with $P, M \in \mathbb{S}^{+}$. Hence Theorem 2 can be applied.

Note that it is possible to find different LMI conditions using the descriptor approach:

Theorem 4: Consider the hybrid system (4), (8)-(11), $S \in \mathbb{R}^{n \times n} \in, \bar{P} \in \mathbb{S}^{+}, L \in \mathbb{R}^{m \times n}$ and the following set of LMIs is verified $\forall i \in\{1, \cdots, M\}$

$$
\mathfrak{S}_{i, j}:=\left(\begin{array}{ccc}
-H e(S) & -S^{\prime}+A_{i} S+M & -S^{\prime}+B_{j} L \\
\star & \left(\sigma^{2}-1\right) \bar{P}+H e\left(A_{i} S\right) & B_{j} L+\bar{P} \\
\star & \star & -\bar{P}
\end{array}\right)<0
$$

If the LMIs (15) is verified, then the set $\mathcal{A}$ defined in (14) is UGpAS with $K=L S$ and $P=S^{\prime-1} \bar{P} S^{-1}$ and solution of (4), (8)-(11) does not posses Zeno behavior.

Proof: Considering the same Lyapunov function as in Theorem 2 Point (i) and (iii) are identical to Theorem 2 as well as the existence of a minimal dwell time.

(ii*) Conditions during flow $\left(\xi \in C_{\mathcal{H}}\right)$ : Note that

$$
\langle\nabla V, f\rangle=x^{\prime} M \dot{x}+\dot{x}^{\prime} M x+\dot{\tau}
$$

Considering the set of matrices $\tilde{G}=(G, G, G), H_{i, j}=\left(-I, A_{i}, B_{j} K\right)$ and writing $H(x)=\sum_{j=1}^{q} \mu_{i}(x) H_{i} \sum_{i=1}^{p} \lambda_{i}(x) H_{i, j}$, one has by definition of $\dot{x}$ that

$$
\left(\dot{x}^{\prime}, x^{\prime}, z^{\prime}\right)\left(H(x)^{\prime} \tilde{G}+\tilde{G}^{\prime} H(x)\right)\left(\dot{x}^{\prime}, x^{\prime}, z^{\prime}\right)^{\prime}=0 .
$$

Adding the previous term to (18) leads to

$$
\langle\nabla V, f\rangle=\left(\dot{x}^{\prime}, x^{\prime}, z^{\prime}\right) \Omega(x)\left(\dot{x}^{\prime}, x^{\prime}, z^{\prime}\right)^{\prime},
$$

with $\Omega(x)=\sum_{j=1}^{q} \mu_{i}(x) \sum_{i=1}^{p} \lambda_{i}(x) \Omega_{i, j}$,

$$
\Omega_{i, j}=\left(\begin{array}{ccc}
-H e(G) & -G+G^{\prime} A_{i}+M & -G+G^{\prime} B_{i, j} K \\
\star & \left(\sigma^{2}-1\right) P+H e\left(A_{i}^{\prime} G\right) & G^{\prime} B_{j} K+P \\
\star & \star & -P
\end{array}\right) .
$$

Notice that $\mathfrak{S}_{i, j}=G^{-1} \Omega_{i, j} G^{\prime-1}$ With $S=G^{-1}, L=K S, P=S^{\prime-1} \bar{P} S^{-1}$.

Since the LMIs (15) are strict, there exists a sufficiently small $\varepsilon>0$ such that

$$
\forall i \in\{1, \cdots, p\}, \Omega_{i, j}<-\varepsilon I \text {. }
$$

Thus by convexity $\forall \xi \in C_{\mathcal{H}}, \forall f \in \mathcal{F}_{\mathcal{H}}(\xi)$,

$$
\langle\nabla V, f\rangle=\left(\dot{x}^{\prime}, x^{\prime}, z^{\prime}\right)^{\prime} \Omega(x)\left(\begin{array}{c}
\dot{x} \\
x \\
z
\end{array}\right) \leq-\varepsilon|\xi|_{\mathcal{A}}^{2},
$$

that is $(5 b)$ is verified. 


\section{EXAMPLES}

A. Linear System

Consider a linear system of the form (1) taken from ([20])

Gain analysis: $A=\left[\begin{array}{cc}0 & 1 \\ -2 & 3\end{array}\right], B=\left[\begin{array}{l}0 \\ 1\end{array}\right], K=[1,-4]$, with $Q=I_{d}$. Solving the LMI conditions (15), one finds an average communication time of $t_{\text {avg }} \simeq 0.25$.

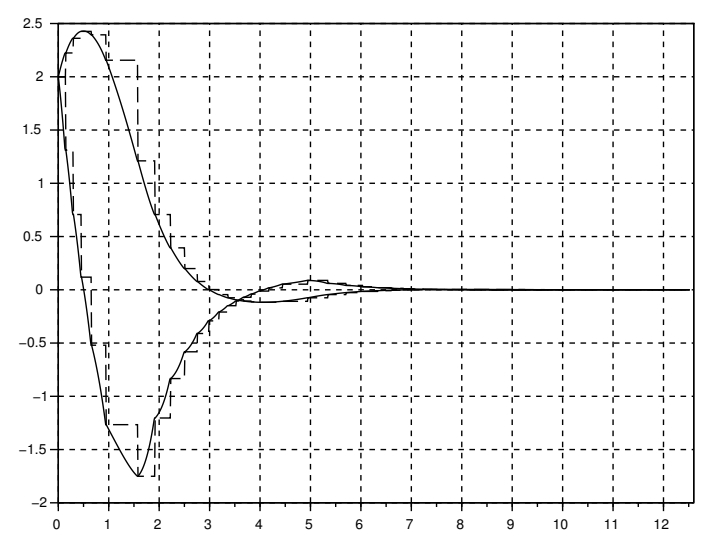

Fig. 1: Gain analysis: Trajectory of the system using $K=1,-4]$, plain $x(t)$ state of the system, dashed sampled value $z(t)$.

Gain synthesis: Considering the same example applying conditions (16).

One obtains an average communication time of $t_{\text {avg }} \simeq 0.4$.

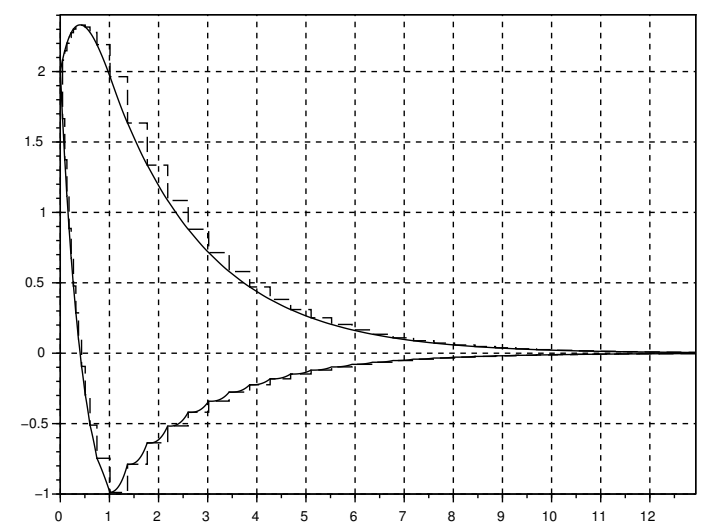

Fig. 2: Gain synthesis: Trajectory of the system using $K=[0.8,-5.3]$, plain $x(t)$ state of the system, dashed sampled value $z(t)$.

Comparison between analysis and synthesis for linear systems: Comparing Fig. 1 and Fig. 2 we observe that circumventing the emulation approach leads to fewer sampling. Furthermore in terms of performance while in Fig. 2 the overshoot is slightly reduced, the convergence rate is lower than to the one observed in 1 begging the question of performance analysis.

\section{B. Non linear system}

Consider the example of a single-link direct-drive manipulator actuated by a permanent magnet DC brush motor [4],[17], with the parameters taken from [17]: 


$$
\dot{x}=\left(\begin{array}{c}
x_{2} \\
-2 \sin \left(x_{1}\right)-3 x_{2}+x_{3} \\
u-x_{2}-x_{3}
\end{array}\right)
$$

Here $i \in\{1,2\}$,

$A_{1}=\left(\begin{array}{ccc}0 & 1 & 0 \\ -2 & -3 & 1 \\ 0 & -1 & -1\end{array}\right), A_{2}=\left(\begin{array}{ccc}0 & 1 & 0 \\ 2 & -3 & 1 \\ 0 & -1 & -1\end{array}\right), B_{1}=B_{2}=(0,0,1)^{\prime}$.

Gain analysis: Using the continuous time model and by using standard $\mathrm{LMI}^{3}$ techniques one finds out $K=$ $[-44,-25,-7.5]$, with $\sigma=0.03$ The trajectory of the system with those parameters is shown in Fig. 3 with an average communication $t_{\text {avg }} \simeq 0.033$.

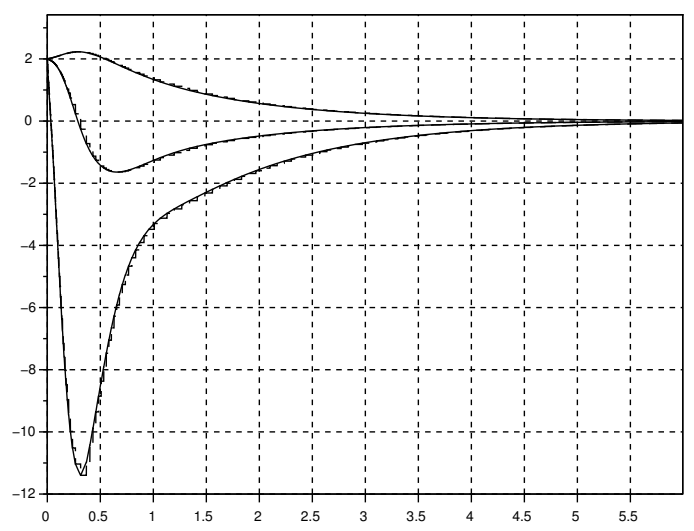

Fig. 3: Gain analysis: Trajectory of the system using $K=[-44,-25,-7.5]$, plain $x(t)$ state of the system, dashed sampled value $z(t)$.

Gain synthesis: Considering the same example applying conditions (16).

With $\sigma=0.3$ one obtains $K=(-12.42182,-3.0724397,-2.270608)$ The trajectory of the system with those parameters is shown in Fig. 4 with an average communication $t_{a v g} \simeq 0.25$.

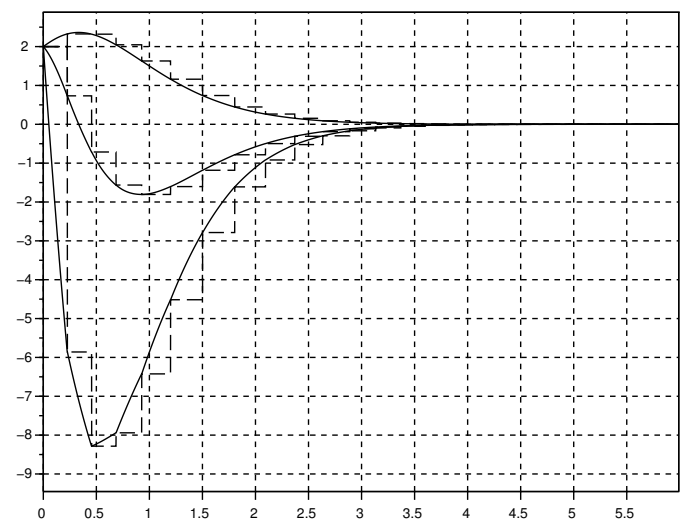

Fig. 4: Gain synthesis: Trajectory of the system using $K=(-12.42182,-3.0724397,-2.270608)$, plain $x(t)$ state of the system, dashed sampled value $z(t)$.

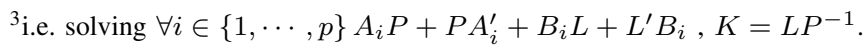


With respect to the analysis case (Fig. 3) it is possible to find bigger values of $\sigma$ while ensuring stability. In this case, the average communication time is also much higher. Furthermore in Fig. 4 the overshoot is significantly reduced when compared to Fig. 3 while the convergence rate remains identical. This observations clearly show the interest of avoiding an emulation based approach in designing control gain for systems with dynamic event triggered sampling.

\section{CONCLUSiON}

In this work new conditions for dynamic event triggered feedback of Lipschitz nonlinear systems have been proposed. The proposed methodology allows to analyze the stability of the system under dynamic event trigger control where an $a$ priori known controller gain is given. Then it is demonstrated how the classic emulation based on the continuous model can be circumvented in order to find a control gain that directly integrate the communication constraints. In both cases the conditions are sufficient and amount to an LMI feasibility problem.

\section{REFERENCES}

[1] K.J. Åstrom, and B. Bernhardsson, Systems with Lebesgue Sampling, Directions in Mathematical Systems Theory and Optimization, pp. 1-13, Springer Berlin, Heidelberg, 2003.

[2] K.J. Åstrom, and B. Wittenmark, Computer Controlled Systems, Prentice Hall, 1997.

[3] C. Briat. Linear parameter-varying and time-delay systems. Analysis, Observation, Filtering \& Control, Springer, 2014.

[4] D. Dawson,J. Carroll and M. Schneider. Integrator back-stepping control of a brush DC motor turning a robotic load.IEEE Transactions on Control Systems Technology Vol 2, No 3,pp 233-244, 1994.

[5] V. S. Dolk, D. P.Borgers \& W. P. M. H. Heemels. Output-Based and Decentralized Dynamic Event-Triggered Control With Guaranteed $\mathcal{L}_{p}$-Gain Performance and zeno-Freeness. IEEE Transactions on Automatic Control, Vol .62 No .1, pp.34 -49.

[6] M.C.F. Donkers, and W.P.M.H. Heemels, Output-Based Event-Triggered Control with Guaranteed $\mathcal{L}_{\infty}$-Gain and Improved Event-Triggering, Proceedings of the $49^{\text {th }}$ Conference on Decision and Control, pp. 3246-3251, 2010.

[7] L. Etienne, S. Di Gennaro \& J.P. Barbot. Event-triggered observers and observer-based controllers for a class of nonlinear systems. In American Control Conference, pp. 4717-4722, 2015.

[8] C. Fiter, L. Hetel, W. Perruquetti, and J-P. Richard A state dependent sampling for linear state feedback Automatica, Vol. 48, No. 8, pp.1860-1867, 2012.

[9] A. Girard. Dynamic triggering mechanisms for event-triggered control. IEEE Transactions on Automatic Control, Vol.60, No.7, pp.1992-1997, 2015.

[10] R. Goebel, R.G. Sanfelice, and A.R. Teel. Hybrid Dynamical Systems: modeling, stability, and robustness. Princeton University Press, 2012.

[11] W.P.M.H. Heemels, J.H. Sandee, and P.P.J. Van Den Bosch, Analysis of Event-Driven Controllers for Linear Systems, International Journal of Control, Vol. 81, No. 4, pp. 571-590, 2008.

[12] W.P.M.H. Heemels, K.H. Johansson, and P. Tabuada, An Introduction to Event-Triggered and Self-Triggered Control. Proceedings of the 51 Conference on Decision and Control, pp. 3270-3285), 2012.

[13] W.P.M.H. Heemels, M.C.F. Donkers, and A.R. Teel, Periodic Event-Triggered Control for Linear Systems, IEEE Transactions of Automatic Control, Vol. 58, No. 4, pp. 847-861, 2013.

[14] W.P.M.H. Heemels, R. Postoyan, M.C.F. Donkers, A.R. Teel, A. Anta, P. Tabuada, and D. Nesic, Periodic Event-Triggered Control, in 'Event-Based Control and Signal Processing', M. Miskowicz Ed., CRC Press/Taylor \& Francis, 2015.

[15] L. Hetel, C. Fiter, H. Omran, A. Seuret, E. Fridman, J.P. Richard and S.I. Niculescu. Recent developments on the stability of systems with aperiodic sampling: an overview. Automatica, Vol. 76, pp. 309-335, 2017.

[16] J. Lunze, and D. Lehmann, A State-Feedback Approach to Event-Based Control, Automatica, Vol. 46, No. 1, pp. 211-215, 2010.

[17] F. Mazenc, V. Andrieu, and M. Malisoff, Design of Continuous-discrete Observers for time-varying nonlinear systems, Automatica, Vol. 57, pp.135$144,2015$.

[18] R. Postoyan, A. Anta, W. P. M. H. Heemels, P. Tabuada, and D. Nesic, Periodic Event-Triggered Control for Nonlinear Systems, Proceedings of the $52^{\text {nd }}$ IEEE Conference on Decision and Control, pp. 7397-7402, 2013.

[19] R. Postoyan, A. Girard, . Triggering mechanism using freely selected sensors for linear time-invariant systems. In IEEE 54th Annual Conference on Decision and Control, pp.4812-4817, 2015.

[20] P. Tabuada. Event-triggered real-time scheduling of stabilizing control tasks. IEEE Transactions on Automatic Control, Vol. 52, No. 9, pp. 1680-1685, 2007.

[21] P. Tallapragada, and N. Chopra, Event-Triggered Dynamic Output Feedback Control of LTI Systems over Sensor-Controller-Actuator Networks, Proceedings of the $52^{\text {nd }}$ Conference on Decision and Control, pp. 4625-4630, 2013.

[22] X. Wang, and M.D. Lemmon, Event-Triggering in Distributed Networked Control Systems, IEEE Transactions on Automatic Control, Vol. 56, No. 3, pp. 586-601, 2011. 